\title{
A general method for retrieving thermal deformation properties of microencapsulated phase change materials or other particulate inclusions in cementitious composites
}

\author{
Benjamin A. Young ${ }^{\mathrm{a}}$, Zhenhua Wei ${ }^{\mathrm{b}}$, Jose Rubalcava-Cruz ${ }^{\mathrm{a}}$, Gabriel Falzone ${ }^{\mathrm{b}}$, Aditya Kumar ${ }^{\mathrm{b}}$, \\ Narayanan Neithalath ${ }^{\mathrm{c}}$, Gaurav Sant ${ }^{\mathrm{b}, \mathrm{d}, \mathrm{e}, *}$, Laurent Pilon ${ }^{\mathrm{a}, "}$ \\ a Department of Mechanical and Aerospace Engineering, Henry Samueli School of Engineering and Applied Science, University of California, Los Angeles, CA 90095, \\ United States \\ ${ }^{\mathbf{b}}$ Laboratory for the Chemistry of Construction Materials (LC2), Department of Civil and Environmental Engineering, Henry Samueli School of Engineering and Applied \\ Science, University of California, Los Angeles, CA 90095, United States \\ ${ }^{c}$ School of Sustainable Engineering and the Built-Environment, Arizona State University, Tempe, AZ 85281, United States \\ d California Nanosystems Institute, University of California, Los Angeles, CA, 90095, United States \\ e Department of Materials Science and Engineering, University of California, Los Angeles, CA, 90095, United States
}

\section{A R T I C L E I N F O}

\section{Keywords:}

Effective medium approximations

Micromechanical modeling

Property measurement

Phase change materials

Construction materials

Cementitious composites

\begin{abstract}
A B S T R A C T
This study examined the effects of spherical core-shell particle inclusions, such as microencapsulated phase change materials (PCMs), on the thermal deformation behavior of cement-based composites. First, simulations of volumetric thermal deformation in representative microstructures were carried out, based on the finite element method (FEM), to predict the effective thermal deformation coefficient of the composites. Excellent agreement was found between the effective thermal deformation coefficient predicted by FEM and by the effective medium approximation (EMA) developed by Schapery (1968). Furthermore, the effective thermal deformation coefficient of cementitious composites with either microencapsulated PCM or quartz particulates was measured. The measured effective thermal deformation coefficients together with Schapery's model were used to retrieve the thermal deformation coefficients of the inclusions themselves. The thermal deformation coefficient of PCM microcapsules was estimated to be similar to that of the shell component due to partial filling of the microcapsules. The results show a means for (i) retrieving the thermal deformation properties of functional core-shell inclusions and (ii) for designing cementitious composites with PCMs which find use in the built environment and high-performance composites.
\end{abstract}

\section{Introduction}

In 2013, The American Society of Civil Engineers gave the road infrastructure in the United States a grade of "D", and estimated that $\$ 67$ billion is spent annually on the repair of deficient or damaged road pavements [1]. Substantial damage is caused to concrete pavements due to volume change that results from temperature change-caused by (i) cement hydration reactions at early ages, over the first 7 days following concrete placement, and (ii) ambient temperature change, at later ages (that results in fatigue damage) [2,3]. Microencapsulated phase change materials (PCMs) have been proposed as a means to mitigate thermal damage in concrete pavements [4,5]. Microencapsulated PCMs, a core-shell particulate, are thermal energy storage materials that can store and release latent heat associated with reversible phase transitions between the liquid and solid phases [6]. In concrete pavements, such storage and release of heat can be exploited to: (i) reduce early-age temperature rise and (ii) decrease the amplitude of diurnal temperature oscillations to reduce thermal fatigue damage $[4,5]$.

PCM particulates with a median diameter on the order of 10 to 20 $\mu \mathrm{m}$ are often produced by an interfacial polymerization process wherein a polymer shell (e.g., of melamine-formaldehyde) is used to encapsulate a core material (e.g., alkanes such as paraffin wax). To provide stressrelief over multiple phase change cycles, typically, the PCM microcapsules are only partially filled-as a result, they contain some internal porosity [6]. Due to the presence of this internal porosity, and their small size, it is challenging to characterize the material properties of these core-shell structures. This is especially so in the

\footnotetext{
"Corresponding authors.

E-mail addresses: gsant@ucla.edu (G. Sant), pilon@seas.ucla.edu (L. Pilon).
} 


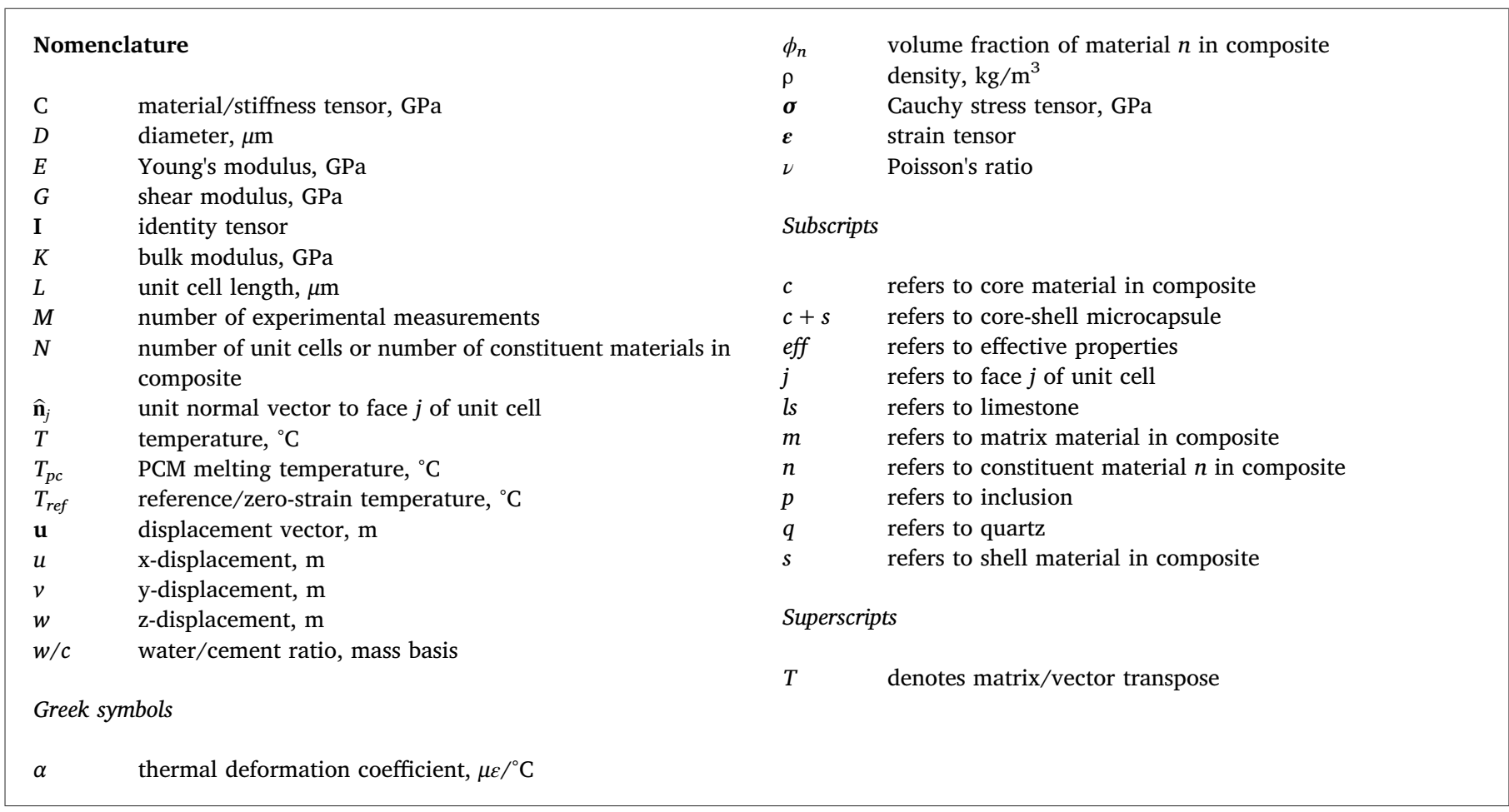

context of thermal deformation behavior when both the core and the shell would expand (or contract), albeit to different extents. This is an issue in applications such as concrete pavements, where it is important to know the thermal deformation coefficients of inclusions that are embedded in the cementitious matrix so as to quantify their influences on the volume stability of the overall solid.

The present study aims to assess the influences of PCM microcapsules on the effective thermal deformation coefficient of cementitious composites by complementary approaches including (i) finite element simulations of volumetric thermal deformation in representative microstructures, (ii) effective medium approximations, and (iii) measurements of linear thermal deformation of prismatic composite specimens. By identifying an effective medium approximation (EMA) capable of accurately estimating the effective thermal deformation coefficient of multicomponent composites consisting of a matrix and core-shell inclusions, a general approach is highlighted (i) for retrieving the thermal deformation coefficient of core-shell microcapsules or other particulate inclusions embedded in a continuous matrix and (ii) for designing cementitious composites with PCMs.

\section{Background}

\subsection{Thermal deformation of solids}

The constitutive law for a linearly elastic material considering thermal effects is given by [7],

$\boldsymbol{\sigma}=\mathbf{C}:\left(\boldsymbol{\varepsilon}-\boldsymbol{\varepsilon}_{\boldsymbol{T}}\right) \quad$ or $\quad \sigma_{i j}=C_{i j k l}\left(\varepsilon_{k l}-\varepsilon_{T, k l}\right)$

where $\boldsymbol{\varepsilon}$ and $\boldsymbol{\varepsilon}_{T}$ denote the total and thermal strain tensors, respectively, and $\mathbf{C}$ is the stiffness tensor. If the material is isotropic, the components of the stiffness tensor $\mathbf{C}$ can be expressed in terms of the material's elastic constants according to [8],

$C_{i j k l}=\lambda \delta_{i j} \delta_{k l}+G\left(\delta_{i k} \delta_{j l}+\delta_{i l} \delta_{j k}\right)$

where the Lamé parameter $\lambda$ and shear modulus $G$ are related to the Young's modulus $E$ and Poisson's ratio $\nu$ according to [8],

$\lambda=\frac{E \nu}{(1+\nu)(1-2 \nu)} \quad$ and $\quad G=\frac{E}{2(1+\nu)}$.
For an isotropic material, the thermal strain $\boldsymbol{\varepsilon}_{T}$ is related to the imposed temperature change $\Delta T$ according to [7],

$\boldsymbol{\varepsilon}_{T}=(\alpha \Delta T) \mathbf{I} \quad$ or $\quad \varepsilon_{T, i j}=\alpha \Delta T \delta_{i j}$

where $\alpha$ is the thermal deformation coefficient and $\Delta T$ is defined with respect to some reference or zero-strain temperature $T_{r e f}$, i.e., $\Delta T=T-T_{\text {ref }}$ [7]. Note that in a homogeneous material that is not mechanically restrained, $\varepsilon=\varepsilon_{T}$ and the stress field is identically zero throughout the material. On the other hand, if it is fully restrained then $\varepsilon=0$ and a stress is induced for $\Delta T \neq 0$.

\subsection{Effective medium approximations}

This study considers three simple effective medium approximations (EMAs) that can be applied to predict the effective thermal deformation coefficient $\alpha_{\text {eff }}$ of composites consisting of two or more constituents. The parallel model, also known as the rule-of-mixtures (ROM) [9], can be used to estimate the effective thermal deformation coefficient of a composite material with $N$ components as a simple volume-weighted average over the constituent thermal deformation coefficients, i.e. [9],

$\alpha_{e f f}=\sum_{n=1}^{N} \phi_{n} \alpha_{n}$

where $\phi_{n}$ and $\alpha_{n}$ are the volume fraction and thermal deformation coefficient of constituent material $n$, respectively. Turner [10] suggested that the ROM be adjusted to weigh each component $n$ by their respective volume fraction $\phi_{n}$ and bulk modulus $K_{n}$, such that

$\alpha_{e f f}=\frac{\sum_{n=1}^{N} \phi_{n} K_{n} \alpha_{n}}{\sum_{n=1}^{N} \phi_{n} K_{n}}$.

Schapery [11] derived an EMA which gave upper and lower bounds for the effective thermal deformation coefficient $\alpha_{e f f}$ of composites with $N$ components based on energy conservation considerations. In this case, $\alpha_{\text {eff }}$ was expressed as an average of upper and lower bounds such that, 
$\alpha_{e f f}=\bar{\alpha}+\left(\frac{\overline{K \alpha}}{\bar{K}}-\bar{\alpha}\right) \frac{\frac{1}{K_{L}}-\frac{1}{K_{e f f}}}{\frac{1}{K_{L}}-\frac{1}{\bar{K}}} \pm \Delta \alpha$

Here, the overline denotes a volume-weighted average over the constituents, i.e. [11],

$\bar{\alpha}=\sum_{n=1}^{N} \phi_{n} \alpha_{n}, \quad \bar{K}=\sum_{n=1}^{N} \phi_{n} K_{n}, \quad$ and $\quad \overline{K \alpha}=\sum_{n=1}^{N} \phi_{n} K_{n} \alpha_{n}$.

In addition, $K_{L}$ is given by,

$K_{L}=\left(\sum_{n=1}^{N} \frac{\phi_{n}}{K_{n}}\right)^{-1}$

and the effective bulk modulus $K_{\text {eff }}$ can be estimated as the average of $K_{L}$ and $\bar{K}$, i.e., $K_{\text {eff }}=\left(K_{L}+\bar{K}\right) / 2$. Finally, the deviation $\Delta \alpha$ in $\alpha_{\text {eff }}$ from the average was expressed as [11],

$$
\begin{gathered}
\Delta \alpha=\frac{\left(\frac{1}{K_{e f f}}-\frac{1}{\bar{K}}\right)^{1 / 2}\left(\frac{1}{K_{L}}-\frac{1}{K_{e f f}}\right)^{1 / 2}}{\frac{1}{K_{L}}-\frac{1}{\bar{K}}} \\
\times\left[\left(\overline{K \alpha^{2}}-\frac{(\overline{K \alpha})^{2}}{\bar{K}}\right)\left(\frac{1}{K_{L}}-\frac{1}{\bar{K}}\right)-\left(\frac{\overline{K \alpha}}{\bar{K}}-\bar{\alpha}\right)^{2}\right]^{1 / 2} .
\end{gathered}
$$

Note that each of the aforementioned EMAs was developed for a linearly elastic composite featuring isotropic constituent materials with arbitrary geometry. The effective thermal deformation coefficient predicted by each EMA depends only on the volume fractions and thermomechanical properties of the constituent materials but is independent of their spatial distribution. They can also be extended to a composite made of any number of constituents. As such, these EMAs are easily applied to cementitious and other composite materials with more than one type of inclusion, including both fine aggregates (e.g., quartz sand) and soft inclusions (e.g., microencapsulated PCM), as well as coarse aggregates that would be present in a typical concrete pavement. In addition, several other EMAs have been developed specifically for two-component composites-further details can be found in Ref. [12].

\section{Materials and methods}

\subsection{Materials}

An ASTM C150 [13] compliant Type I/II ordinary portland cement (OPC) was mixed with deionized (DI) water to prepare cement pastes (matrix only) and mortars (matrix + inclusions) in accordance with ASTM C192 [14]. The OPC had a nominal mass-based mineralogical composition of: $56.5 \% \mathrm{Ca}_{3} \mathrm{SiO}_{5}, 18.0 \% \mathrm{Ca}_{2} \mathrm{SiO}_{4}, 11.4 \% \mathrm{Ca}_{4} \mathrm{Al}_{2} \mathrm{Fe}_{2} \mathrm{O}_{10}$, $6.3 \% \mathrm{Ca}_{3} \mathrm{Al}_{2} \mathrm{O}_{6}, 4.6 \% \mathrm{CaCO}_{3}$, and $1.1 \% \mathrm{CaSO}_{4} \cdot 2 \mathrm{H}_{2} \mathrm{O}$. ASTM C778 [15] compliant graded quartz sand and microencapsulated PCM inclusions (MPCM24D, Microtek Laboratories) were used as inclusions within the cement mortars. The microencapsulated PCMs consisted of a paraffin core encapsulated within a melamine-formaldehyde (MF) shell. The microencapsulated PCMs were received in the form of dry powders. The corresponding peak melting temperature and enthalpy of phase change of microencapsulated PCMs were $27.8^{\circ} \mathrm{C}$ and $161.2 \pm 0.5 \mathrm{~kJ} / \mathrm{kg}$ representing the average value over three melting and solidification cycles.

Cementitious mortars were prepared with various volume fractions (i.e., dosages) of microencapsulated PCM and/or quartz inclusions at a water-to-cement ratio (w/c, mass basis) of 0.45 . For mortars containing microencapsulated PCM inclusions only, the inclusions were dosed for three different volume fractions, namely, 5, 10 and 20 vol.\%. For mortars containing quartz inclusions only, the quartz dosages were 10 , 20 , and 50 vol.\%. For mortars containing mixed inclusions, the total inclusion volume fraction was fixed at 50 vol.\%, where the microencapsulated PCM inclusions comprised 10, 15, or 20 vol.\%. The microencapsulated PCMs were mixed with the anhydrous OPC by hand prior to the addition of DI water to ensure random and uniform distribution in the mortar. To enhance the fluidity of the fresh mixtures, a commercially available water-reducing admixture (MasterGlenium 7500 , BASF Corporation) was added at a dosage on the order of 0-to1.5 wt.\% by mass of cement, depending on the type and dosage of inclusions present.

\subsection{Experimental methods}

Thermal deformation coefficient measurements were carried out using prismatic specimens with various mixture proportions, as described above. The specimens were cast in $2.54 \mathrm{~cm} \mathrm{x} 2.54 \mathrm{~cm} \mathrm{x}$ $28.50 \mathrm{~cm}$ molds in accordance with ASTM C157 [16] and cured under $100 \%$ relative humidity for the first $24 \mathrm{~h}$ at $25^{\circ} \mathrm{C}$. Following demolding after $24 \mathrm{~h}$, the specimens were sealed with aluminum tape and cured in sealed bags at $25^{\circ} \mathrm{C}$ for 28 days. Then, the length of each specimen was measured at $25^{\circ} \mathrm{C}$ and the specimens were transferred to an environmental chamber (KB024-DA, Darwin Chambers Company) at $45^{\circ} \mathrm{C}$, and stored for $2 \mathrm{~h}$ until they reached thermal equilibrium. The fractional length change (i.e., the linear thermal strain) $\Delta L / L_{0}$ due to temperature change $\Delta T=45^{\circ} \mathrm{C}-25^{\circ} \mathrm{C}$ was measured with a length comparator, as illustrated in Fig. 1. Then, the thermal deformation coefficient of the specimens was calculated according to,

$\alpha_{e f f}=\frac{\Delta L}{L_{0} \Delta T}$.

In addition, elastic thermal strain within the specimens was verified by also calculating $\alpha_{e f f}$ based on a temperature change from $5^{\circ} \mathrm{C}$ to $45^{\circ} \mathrm{C}$ and verifying that the measured $\alpha_{\text {eff }}$ was independent of the imposed temperature change. It was also confirmed that the specimens recovered their initial length when returned to their initial temperature of $25^{\circ} \mathrm{C}$. Such elastic stress-strain behavior implies that the thermal deformation coefficient of cementitious composites would remain constant across the range of diurnal temperature variations that would be experienced by a concrete pavement. However, when the concrete's temperature falls below $0^{\circ} \mathrm{C}$, its thermomechanical behavior is

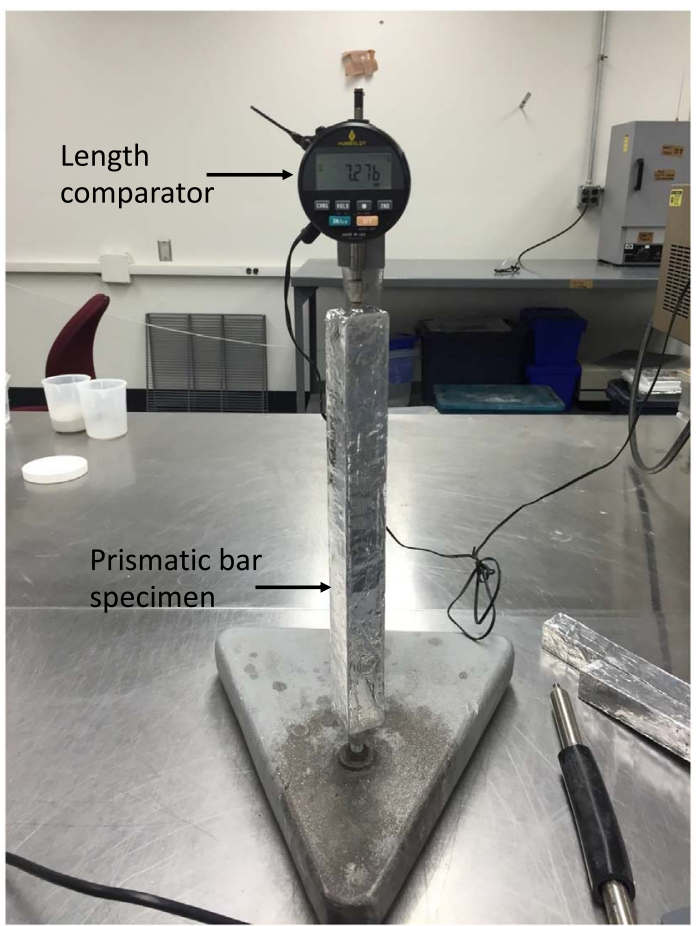

Fig. 1. Photograph of the experimental setup used to measure the effective thermal deformation coefficient of cementitious composites containing microencapsulated PCMs and/or quartz sand inclusions. 
complicated by melting and freezing of water within its pores [17] and thus cannot be treated as simple elastic deformation.

\section{Analysis}

\subsection{Schematic and assumptions}

Fig. 2 illustrates the computational cubic unit cells of length $L$ utilized in this study, along with the associated coordinate system. Free thermal deformation was simulated numerically in domains with ordered monodisperse packing arrangements, namely (a) body-centered cubic (BCC), (b) simple cubic (SC), or (c) face-centered cubic (FCC) or in domains with randomly distributed (d) monodisperse or (e) polydisperse microcapsules. The core and shell volume fractions $\phi_{c}$ and $\phi_{s}$ can be expressed in terms of the average core and shell diameters $\overline{D_{c}}$ and $\overline{D_{s}}$ and the unit cell length $L$ according to,

$\phi_{c}=\frac{N_{p} \pi \bar{D}_{c}^{3}}{6 L^{3}}$ and $\phi_{s}=\frac{N_{p} \pi\left(\bar{D}_{s}^{3}-\bar{D}_{c}^{3}\right)}{6 L^{3}}$

where $N_{p}$ is the number of core-shell particles contained within the unit cell.

A stochastic packing algorithm [18] was used to generate the size and location of randomly distributed monodisperse and polydisperse microcapsules within unit cells of edge length $L=75 \mu \mathrm{m}$ (Fig. 2d-e). The algorithm placed microcapsules at random locations within the unit cell until the desired core volume fraction $\phi_{c}$ was achieved within $0.5 \%$. It ensured that the minimum centroidal distance between two sphere centers $C_{D}$ was greater than the sum of their radii $r_{1}+r_{2}$ [18]. The packing algorithm considered a particle size distribution with average shell diameter $\bar{D}_{s}=18 \mu \mathrm{m}$ as well as 10th and 95th percentile shell diameters equal to $9 \mu \mathrm{m}$ and $33 \mu \mathrm{m}$, respectively [18]. All microcapsules had a shell thickness of $1 \mu$ m, i.e., $\left(D_{s}-D_{c}\right) / 2=1$ $\mu \mathrm{m}$ [18]. Previously, we used the same microstructures to predict the effective thermal conductivity [19] and elastic moduli [20] of threecomponent composites. The size and location of the microcapsules can be found in supplementary material.

To make the problem mathematically tractable, it was assumed that (i) all materials were linearly elastic and isotropic, (ii) body forces were negligible, and (iii) continuous contact was maintained at the material interfaces, i.e., no sliding or gapping was allowed.

\subsection{Governing equations and boundary conditions}

The stress field in each component was governed by the steady-state differential equilibrium equation [8],

$\nabla \cdot \sigma_{n}=0$

where $\sigma_{n}$ is the local stress tensor in component $n$ referring to the core (subscript $c$ ), shell (subscript $s$ ), or matrix (subscript $m$ ). The stress $\boldsymbol{\sigma}_{n}$ and strain $\boldsymbol{\varepsilon}_{n}$ tensors were related according to the constitutive law given by Eq. (1). Here, the temperature rise $\Delta T$ was imposed by fixing the temperature at each face of the cube at $T=T_{\text {ref }}+\Delta T$ such that solving the steady-state heat diffusion equation resulted in a spatially uniform temperature $T$ throughout the domain. Additionally, the straindisplacement relation in each component was given by [8],

$\boldsymbol{\varepsilon}_{n}=\frac{1}{2}\left[\nabla \mathbf{u}_{n}+\left(\nabla \mathbf{u}_{n}\right)^{T}\right]$

where $\boldsymbol{u}_{n}=\left[u_{n}, v_{n}, w_{n}\right]^{T}$ is the displacement vector in component $n$.

In order to fully define the problem, boundary conditions on each of the cube's six faces, denoted by index $j$, were prescribed to represent free volumetric thermal deformation. For ordered packing arrangements (Fig. 2a-c), the faces at $x=0(j=1), y=0(j=2)$, and $z=0(j$ $=3$ ) were fixed by virtue of symmetry, i.e.,

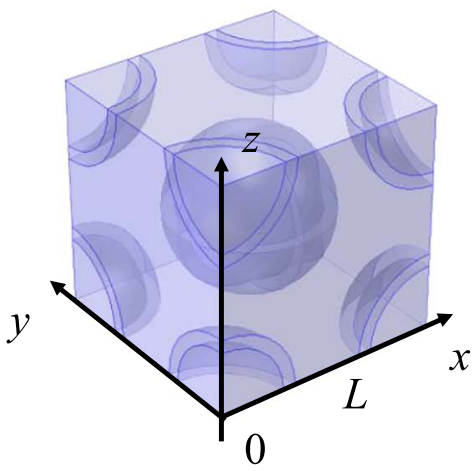

(a) $\mathrm{BCC}$

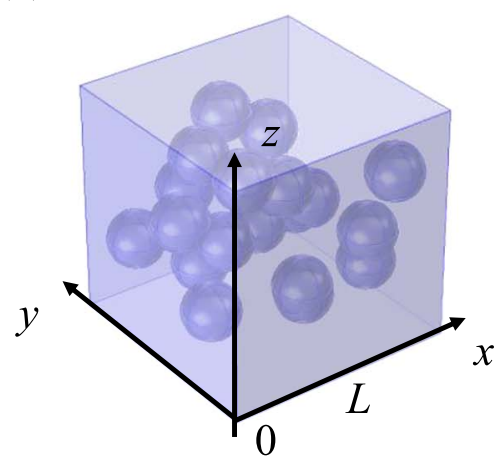

(d) Random monodisperse

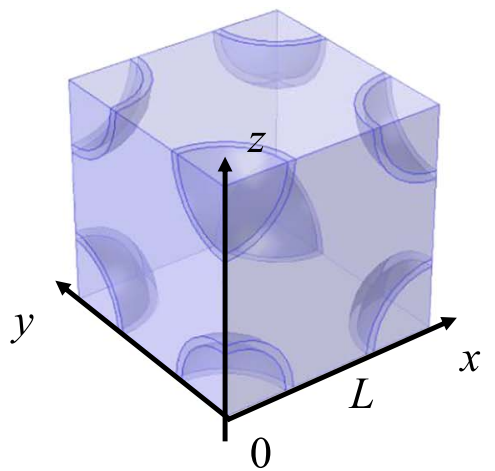

(b) $\mathrm{SC}$

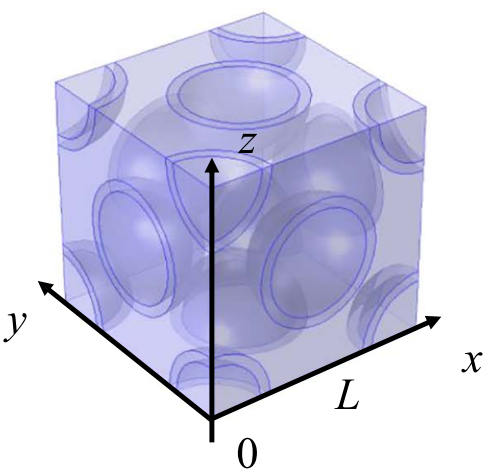

(c) FCC

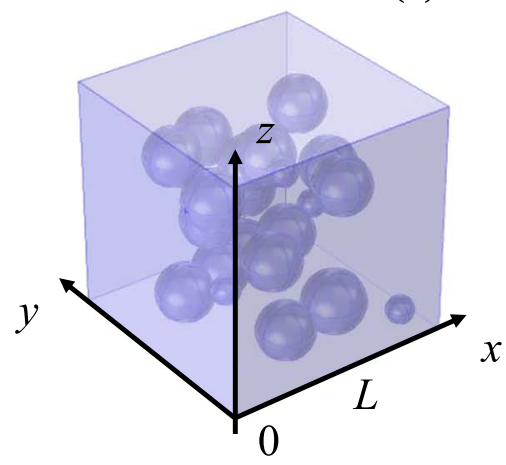

(e) Random polydisperse

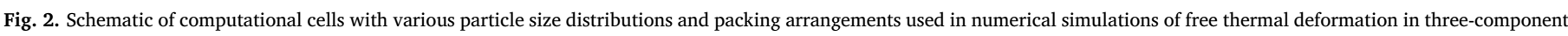

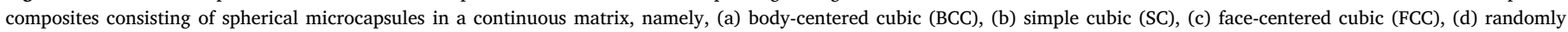
distributed monodisperse microcapsules, and (e) randomly distributed polydisperse microcapsules. 
$\mathbf{u}_{n} \cdot \widehat{\mathbf{n}}_{j}=0 \quad$ for $\quad j=1,2$, or 3

where $\widehat{\mathbf{n}}_{j}$ denotes the unit normal vector to face $j$. The remaining faces were allowed to expand freely. As such, the traction force on these faces vanished [8],

$\boldsymbol{\sigma}_{n} \cdot \hat{\mathbf{n}}_{j}=0$ for $j=4,5$, or6.

Moreover, for unit cells with randomly distributed microcapsules (Fig. 2d-e), all six faces were allowed to expand freely, i.e.,

$\boldsymbol{\sigma}_{n} \cdot \hat{\mathbf{n}}_{j}=0$ for $j=1,2, \ldots$, or6.

\subsection{Data processing}

Solving the governing Eqs. (1), (13) and (14) along with their corresponding boundary conditions yields the displacement field $\mathbf{u}_{n}(x, y, z)$ in each component of the composite volume initially at $V_{0}$. Then, the corresponding change in volume $\Delta V$ of the deformed body for a temperature change of $\Delta T$ was used to compute the effective thermal deformation coefficient $\alpha_{\text {eff }}$ (in $\mu \varepsilon /{ }^{\circ} \mathrm{C}$, neglecting higher-order strain terms) according to [7],

$\alpha_{e f f}=\frac{1}{3} \frac{\Delta V}{V_{0} \Delta T}$.

\subsection{Method of solution}

The governing Eqs. (1), (13), and (14) along with the corresponding boundary conditions [Eqs. (15)- (17)] were solved using the finite element method with the commercially available finite element solver COMSOL Multiphysics . Computation was performed on a single CPU with two $2.6 \mathrm{GHz}$ processors and 8 GB RAM. Numerical convergence was verified such that the predicted value of $\alpha_{e f f}$ did not change by more than $0.5 \%$ when the minimum element size was decreased by a factor of 2. Numerical convergence was reached using a minimum element size of $0.2 \mu \mathrm{m}$ and a maximum element growth rate of 1.5. The number of finite elements in the computational domain ranged from 66,252 to 534,108 . In addition, it was verified that the predicted $\alpha_{e f f}$ was independent of the imposed temperature difference $\Delta T$ for $5^{\circ} \mathrm{C}<\Delta T$ $<30^{\circ} \mathrm{C}$.

\section{Results and discussion}

\subsection{Numerical simulations}

This section uses numerical simulations of free thermal deformation to elucidate the effect of geometric parameters as well as constituent material properties on the effective thermal deformation coefficient $\alpha_{\text {eff }}$ of three-component composites consisting of spherical microcapsules in a continuous matrix. Table 1 outlines the baseline case values of thermal deformation coefficient $\alpha$, Young's modulus $E$, Poisson's ratio $\nu$, and bulk modulus $K$ used in the simulations. These properties were chosen to represent a composite consisting of microencapsulated PCM inclusions in a cementitious matrix [20-23]. Note that the bulk modulus $K$ is related to the Young's modulus $E$ and Poisson's ratio $\nu$ according to [8],

$K=\frac{E}{3(1-2 \nu)}$.

\subsubsection{Effect of computational domain size}

Fig. 3 plots the numerically predicted effective thermal deformation coefficient $\alpha_{e f f}$ as a function of the total number of simulated BCC, FCC, or SC unit cells $N$ in the cubic computational domain for $N=1,8$ $(2 \times 2 \times 2)$, or $27(3 \times 3 \times 3)$. Here, unit cells with three sets of core and shell volume fractions $\phi_{c}$ and $\phi_{s}$ were simulated. Fig. 3 establishes that $\alpha_{e f f}$ was independent of the number of unit cells simulated. The predicted $\alpha_{\text {eff }}$ was also nearly identical for each ordered packing arrangement (BCC, FCC, or SC). Indeed, the maximum relative difference between $\alpha_{\text {eff }}$ for any two different domain sizes or packing arrangements was less than $2 \%$. Note that these small variations in $\alpha_{\text {eff }}$ were expected due to the absence of periodicity in the imposed boundary conditions. Based on this result, a domain consisting of a single unit cell with BCC packing was used for all further simulations of domains featuring ordered microcapsules.

\subsubsection{Effect of core and shell volume fractions}

Fig. 4 plots the numerically predicted effective thermal deformation coefficient $\alpha_{e f f}$ as a function of the core-shell volume fraction $\phi_{c}$ ${ }_{+s}=\phi_{c}+\phi_{s}$ ranging from 0 to 0.5 . The thermomechanical properties of each constituent material were those of the baseline case (Table 1). Here, the ratio of core to core-shell volume fractions $\phi_{c} / \phi_{c+s}$ was held constant and equal to either 0.5 or 0.85 . The corresponding core and shell volume fractions $\phi_{c}$ and $\phi_{s}$ were imposed by fixing either (i) the core diameter $D_{c}$, (ii) the shell diameter $D_{s}$, or (iii) the unit cell length $L$ in Eq. (12). Fig. 4 shows that the effective thermal deformation coefficient $\alpha_{\text {eff }}$ increased linearly with increasing core-shell volume fraction $\phi_{c+s}$. This was due to the fact that both the core thermal deformation coefficient $\alpha_{c}$ and the shell thermal deformation coefficient $\alpha_{s}$ were larger than that of the matrix $\alpha_{m}$. In addition, for given core and shell volume fractions, $\alpha_{e f f}$ was the same regardless of which geometric parameter $D_{c}, D_{s}$, or $L$ was fixed.

Moreover, Fig. 4 also shows predictions from (i) the ROM or parallel model [Eq. (5)], (ii) Turner's model [Eq. (6)], and (iii) the average of the upper and lower bounds of Schapery's model [Eq. (7)]. Excellent agreement was found between the numerical predictions of $\alpha_{\text {eff }}$ and predictions from Schapery's model. The ROM and Turner's model overestimated and underestimated the numerical predictions, respectively.

\subsubsection{Effect of size and spatial distributions}

Table 2 reports the effective thermal deformation coefficient $\alpha_{\text {eff }}$ predicted numerically using computational domains with different core and shell volume fractions $\phi_{c}$ and $\phi_{s}$ and with either ordered monodisperse or randomly distributed monodisperse or polydisperse microcapsules. Note that very similar values of $\alpha_{\text {eff }}$ were obtained for Cases $1-3\left(\phi_{c} \approx 0.1, \phi_{s} \approx 0.04\right)$ and also for Cases $4-5\left(\phi_{c} \approx 0.2, \phi_{s} \approx 0.05\right)$. Thus, for a given $\phi_{c}$ and $\phi_{s}, \alpha_{\text {eff }}$ did not depend on the packing arrangement (i.e., ordered or random) or microcapsule size distribution (i.e., monodisperse or polydisperse). In other words, a single unit cell with BCC packing was representative of the thermal elastic behavior of cells with randomly distributed monodisperse or polydisperse microcapsules. In light of these results, a computational domain consisting of a single unit cell with BCC packing was utilized for the remainder of this study.

Moreover, predictions from Schapery's model [Eq. (7)] were again in excellent agreement with the numerically predicted $\alpha_{\text {eff }}$ and differed by less than $2 \%$ for all cases considered in Table 2 .

\subsubsection{Effect of constituent thermal deformation coefficients}

Fig. 5 plots the numerically predicted effective thermal deformation

\section{Table 1}

Baseline case values of thermal deformation coefficient $\alpha$, Young's modulus $E$, Poisson's ratio $\nu$, and bulk modulus $K$ of the core, shell, and matrix materials used in numerical thermal deformation simulations.

\begin{tabular}{lllllll}
\hline Material & Subscript & $\alpha\left(\mu \varepsilon /{ }^{\circ} \mathrm{C}\right)$ & $E(\mathrm{GPa})$ & $\nu$ & $K(\mathrm{GPa})$ & Ref. \\
\hline Core & $\mathrm{c}$ & 109 & 0.056 & 0.499 & 9.3 & {$[20,21]$} \\
Shell & $\mathrm{s}$ & 50 & 6.3 & 0.34 & 6.6 & {$[20,22]$} \\
Matrix & $\mathrm{m}$ & 11 & 22.1 & 0.15 & 10 & {$[23]$} \\
\hline
\end{tabular}




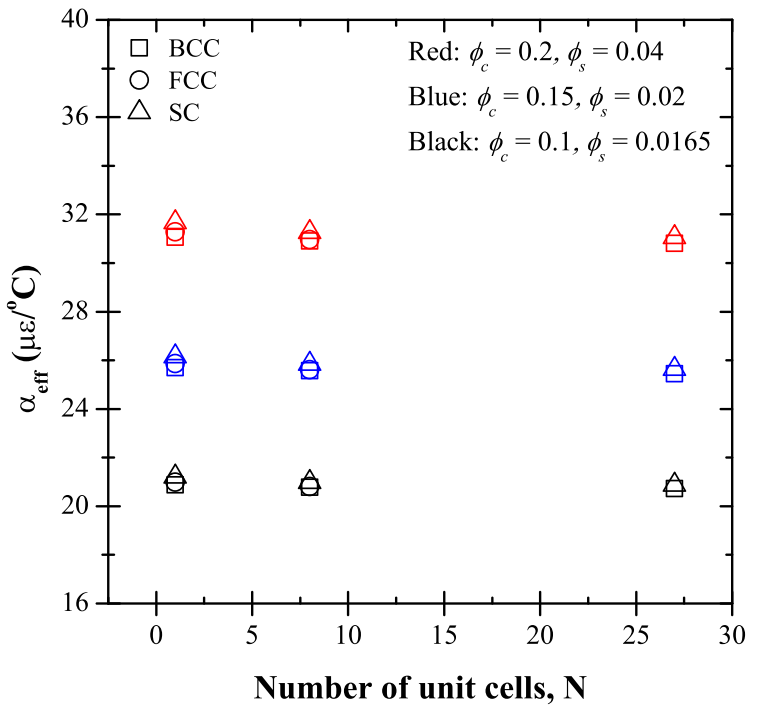

Fig. 3. Effective thermal deformation coefficient $\alpha_{e f f}$ of three-component composites consisting of spherical microcapsules in a continuous matrix predicted numerically as a function of the number $N$ of BCC, FCC, or SC unit cells in the cubic computational domain. The thermomechanical properties of the core, shell, and matrix materials are listed in Table 1.

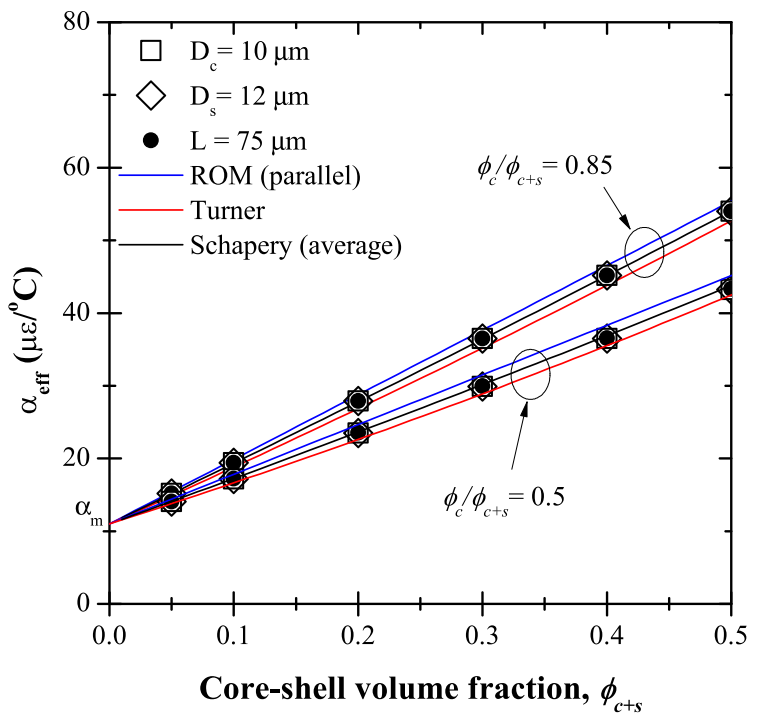

Fig. 4. Numerically predicted effective thermal deformation coefficient $\alpha_{\text {eff }}$ as a function of the core-shell volume fraction $\phi_{c+s}=\phi_{c}+\phi_{s}$ for the baseline case (Table 1 ). Here, the ratio $\phi_{c} / \phi_{c+s}$ was held constant and equal to 0.5 or 0.85 and the corresponding core and shell volume fractions $\phi_{c}$ and $\phi_{s}$ were imposed by fixing either (i) the core diameter $D_{c}$, (ii) the shell diameter $D_{s}$, or (iii) the unit cell length $L$. coefficient $\alpha_{\text {eff }}$ as a function of (a) the core thermal deformation coefficient $\alpha_{c}$, (b) the shell thermal deformation coefficient $\alpha_{s}$, and (c) the matrix thermal deformation coefficient $\alpha_{m}$ ranging from 0 to 500 $\mu \varepsilon /{ }^{\circ} \mathrm{C}$ for core volume fraction $\phi_{c}=0.2$ and shell volume fraction $\phi_{s}=$ 0.15 . Each plot shows two different sets of values $\alpha_{c}, \alpha_{s}$, or $\alpha_{m}$ between 5 and $400 \mu \varepsilon /{ }^{\circ} \mathrm{C}$ in an effort to explore a wide range of variation in constituent thermal deformation coefficients. Fig. 5 shows that $\alpha_{e f f}$ increased linearly with each constituent's thermal deformation coefficient.

Moreover, Fig. 5 shows predictions by the ROM, Turner, and Schapery models. Here also, excellent agreement was observed between the Schapery model and the numerical predictions over the wide range of constituent thermal deformation coefficients considered, with a relative error not exceeding $3 \%$.

\subsection{Experimental measurements and property retrieval}

This section presents a general method to retrieve the thermal deformation coefficient of particulate inclusions that are difficult to measure directly by combining Schapery's model [Eq. (7)], previously validated numerically, with the measured effective thermal deformation coefficient. Here, the thermal deformation coefficient $\alpha_{p}$ of the inclusions was retrieved by least-square fitting the experimentally measured effective thermal deformation coefficient for $\mathrm{M}$ different inclusion volume fractions $\phi_{i}$ to Schapery's model by minimizing the sum-of-squares error $\delta$ given by,

$\delta=\sum_{i=1}^{M}\left[\alpha_{\text {eff }, \text { exp }}\left(\phi_{i}\right)-\alpha_{\text {eff, Schapery }}\left(\phi_{i}\right)\right]^{2}$.

\subsubsection{Validation}

The suggested method for retrieving the inclusion thermal deformation coefficient was validated by demonstrating its use on experimental data presented by Walker et al. [24] for cement pastes with crushed limestone inclusions with volume fraction $\phi_{l s}$ ranging from 0 to 0.6. Additionally, the authors directly measured the thermal deformation coefficient of the cement paste alone as $\alpha_{m}=9.72 \mu \varepsilon /{ }^{\circ} \mathrm{C}$, and that of the limestone as $\alpha_{l s}=4.4 \mu \varepsilon /{ }^{\circ} \mathrm{C}$ by sawing specimens from the parent stone [24]. Here, the elastic modulus and Poisson's ratio of the limestone were taken as $31 \mathrm{GPa}$ and 0.25 , respectively [25]. Fig. 6 plots the experimentally measured effective thermal deformation coefficient $\alpha_{\text {eff }}$ as a function of limestone volume fraction $\phi_{l s}$ [24]. The error bars correspond to an uncertainty of $\pm 5 \%$. Fig. 6 also shows the best fit obtained with the Schapery model, corresponding to a retrieved value of $\alpha_{l s}=4.3 \mu \varepsilon /{ }^{\circ} \mathrm{C}$. This value of $\alpha_{l s}$ agrees very well with that measured directly by the authors, thus demonstrating that our suggested approach can be used to obtain accurate estimates of inclusion thermal expansion coefficients.

\subsubsection{Thermal deformation coefficient of PCM microcapsules}

Fig. $7 \mathrm{a}$ plots the experimentally measured effective thermal deformation coefficient $\alpha_{\text {eff }}$ of cement paste with microencapsulated PCM

Table 2

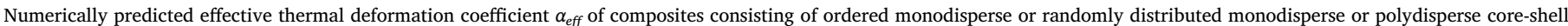
microcapsules in a continuous matrix for the baseline case (Table 1).

\begin{tabular}{|c|c|c|c|c|c|c|c|c|c|}
\hline Case & Packing & Size distribution & $N_{p}$ & $L(\mu \mathrm{m})$ & $\phi_{c}$ & $\phi_{s}$ & $\alpha_{e f f}\left(\mu \varepsilon /{ }^{\circ} \mathrm{C}\right)$ & $\alpha_{e f f}\left(\mu \varepsilon /{ }^{\circ} \mathrm{C}\right)$ & \% Error \\
\hline & & & & & & & Numerical & Schapery [Eq. (7)] & \\
\hline 1 & BCC & Monodisperse & 2 & 22.2 & 0.096 & 0.041 & 21.2 & 21.2 & 0 \\
\hline 2 & Random & Monodisperse & 19 & 75 & 0.097 & 0.041 & 21.0 & 21.3 & 1.5 \\
\hline 3 & Random & Polydisperse & 22 & 75 & 0.095 & 0.041 & 20.9 & 21.1 & 1.1 \\
\hline 4 & BCC & Monodisperse & 2 & 17.4 & 0.2 & 0.046 & 31.3 & 31.2 & 0.2 \\
\hline 5 & Random & Polydisperse & 38 & 75 & 0.2 & 0.046 & 31.7 & 31.2 & 1.4 \\
\hline
\end{tabular}


(a)

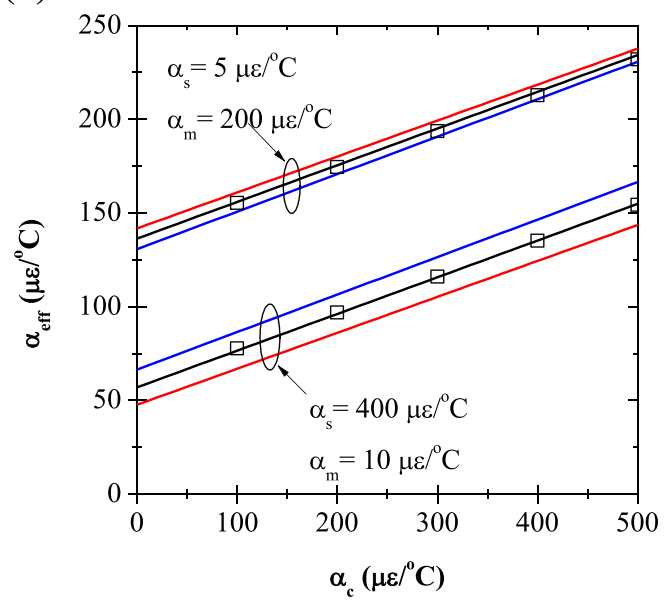

(c)

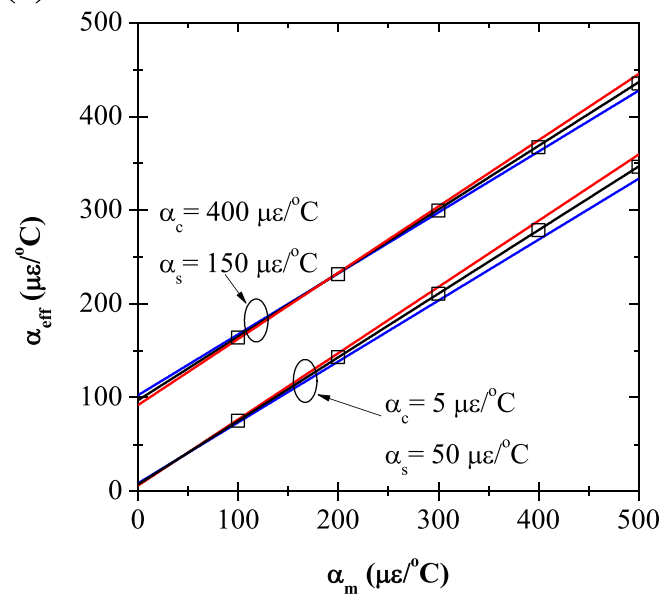

(b)
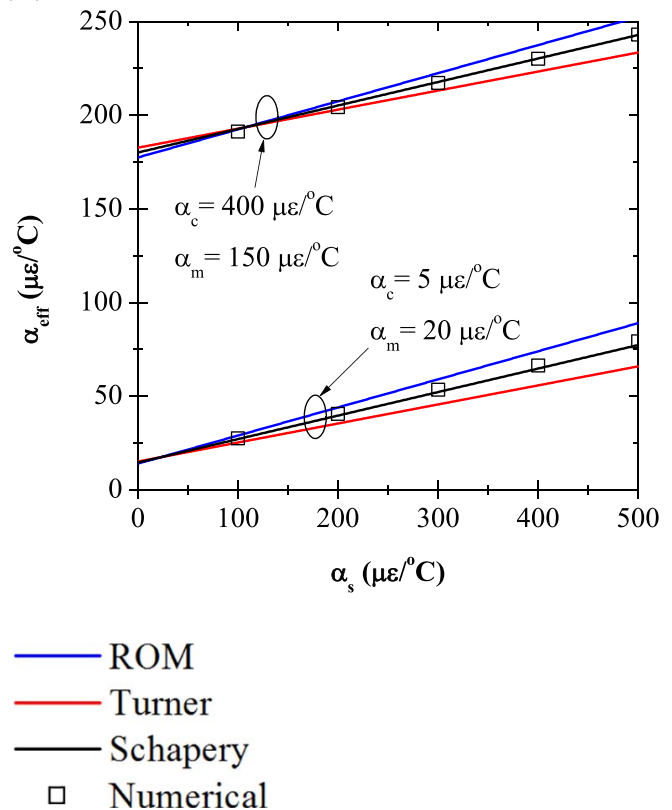

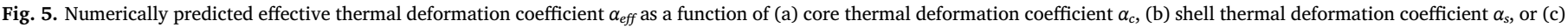
matrix thermal deformation coefficient $\alpha_{m}$ ranging from 0 to $500 \mu \varepsilon /{ }^{\circ} \mathrm{C}$. The core and shell volume fractions were $\phi_{c}=0.2$ and $\phi_{s}=0.15$.

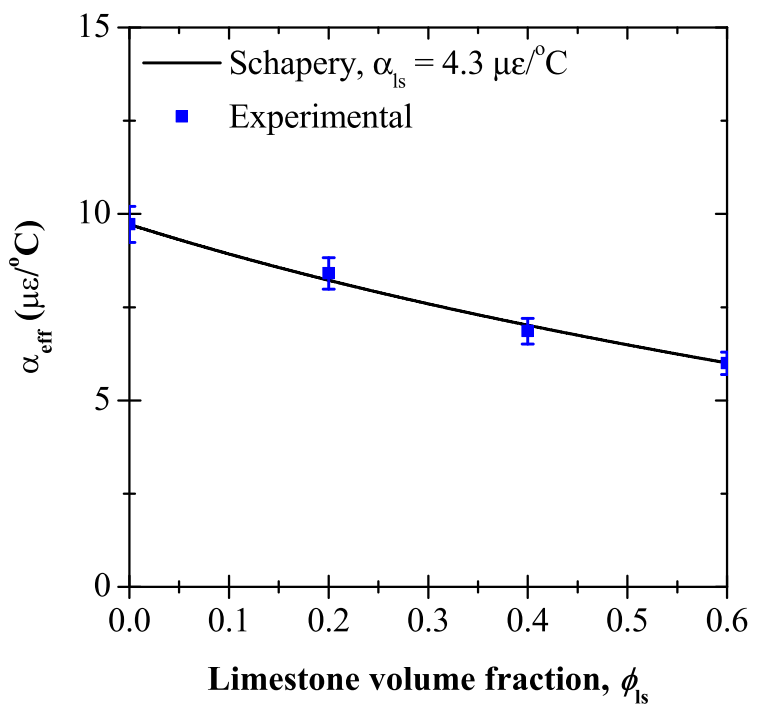

Fig. 6. Effective thermal deformation coefficient $\alpha_{\text {eff }}$ of cement pastes with crushed limestone inclusions as a function of limestone volume fraction $\phi_{l s}$ ranging from 0 to 0.6 measured by Walker et al. [24] and predicted by Schapery's model using the retrieved value of $\alpha_{l s}=4.3 \mu \varepsilon /{ }^{\circ} \mathrm{C}$. specimens as a function of microencapsulated PCM volume fraction $\phi_{c}$ ${ }_{+s}$ ranging from 0 to 0.3 . The error bars correspond to one standard deviation or $68 \%$ confidence interval of three measurements. Fig. $7 \mathrm{a}$ shows that $\alpha_{e f f}$ slightly increased with increasing $\phi_{c+s}$, confirming that the thermal deformation coefficient of the PCM microcapsules was indeed larger than that of the cementitious matrix. The latter was measured to be $\alpha_{m}=14.1 \mu \varepsilon /{ }^{\circ} \mathrm{C}$, corresponding to $\phi_{c+s}=0$. The thermal deformation coefficient $\alpha_{c+s}$ of the PCM microcapsules was retrieved by least-square fitting the experimental measurements of $\alpha_{e f f}$ with Schapery's model, as outlined previously. In this case, the retrieved value of $\alpha_{c+s}$ was $42 \mu \varepsilon /{ }^{\circ} \mathrm{C}$. Interestingly, this value was similar to the thermal deformation coefficient of the MF shell, reported in the literature as $\alpha_{s}=50 \mu \varepsilon /{ }^{\circ} \mathrm{C}$ [22].

The PCM microencapsulation process is often carried out at elevated temperatures around $100^{\circ} \mathrm{C}$ [6]. During subsequent cooling, the paraffinous core contracts more than the shell, i.e., $\alpha_{c}>>\alpha_{s}$ (see Table 1), leaving some empty space in the PCM microcapsules at room temperature. Then, upon subsequent heating, the thermal deformation coefficient $\alpha_{c}$ of the core material has negligible impact on the thermal deformation coefficient $\alpha_{c+s}$ of the core-shell microcapsule, as the core is allowed to expand freely without exerting any stress on the shell. Therefore, the thermal deformation coefficient of the PCM microcapsule $\alpha_{c+s}$ is expected to be similar to that of the shell material for temperatures below $100^{\circ} \mathrm{C}$. 
(a)

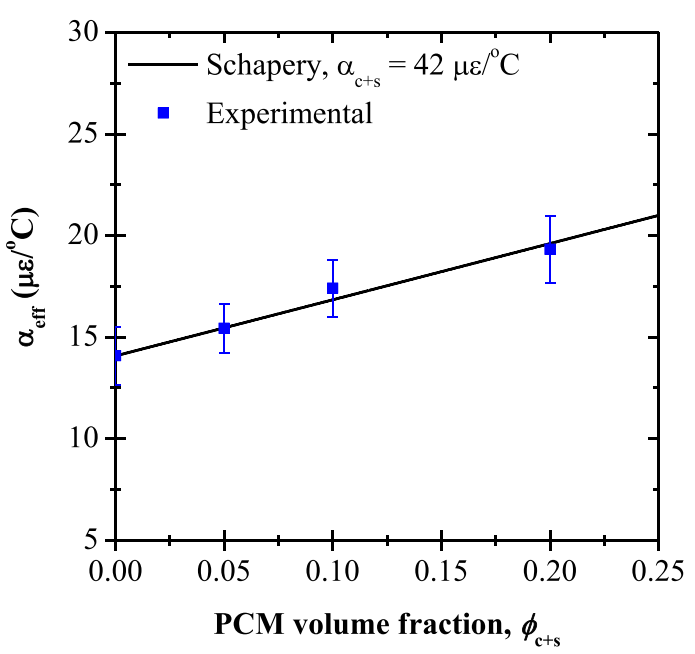

(b)

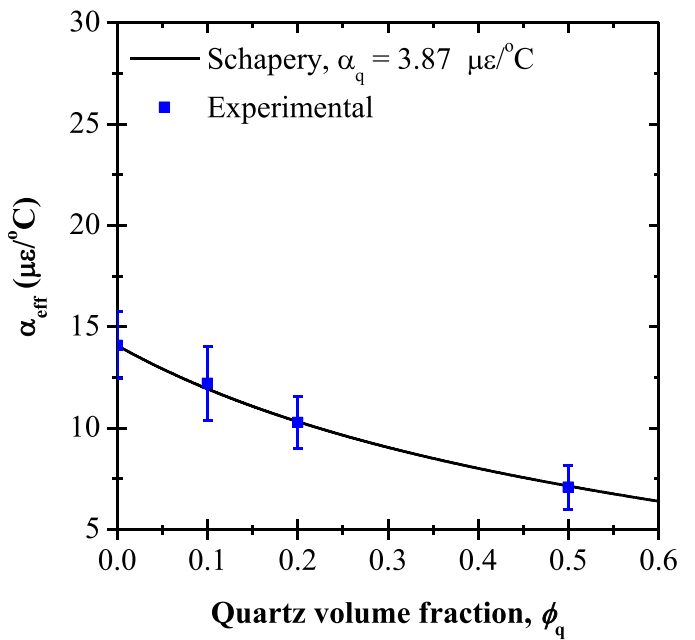

(c)

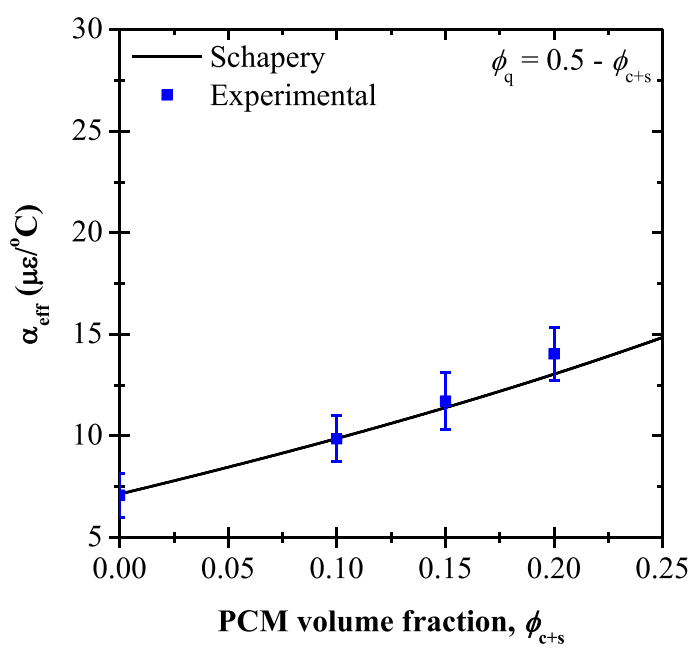

Fig. 7. Experimentally measured thermal deformation coefficient $\alpha_{\text {eff }}$ of cement pastes containing (a) microencapsulated PCM with $\phi_{c+s}$ ranging from 0 to 0.25 , (b) quartz sand with $\phi_{q}$ ranging from 0 to 0.6 , or (c) both microencapsulated PCM and quartz sand with $\phi_{c+s}$ ranging from 0 to 0.25 and $\phi_{q}+\phi_{c+s}=0.5$.

\subsubsection{Thermal deformation coefficient of quartz sand inclusions}

Fig. 7b plots the experimentally measured effective thermal deformation coefficient $\alpha_{e f f}$ of cement mortar (i.e., cement paste with quartz sand inclusions) as a function of quartz inclusion volume fraction $\phi_{q}$ ranging from 0 to 0.5 . In this case, the low thermal deformation coefficient of the quartz sand caused $\alpha_{\text {eff }}$ to decrease with increasing quartz volume fraction $\phi_{q}$. Here again, the thermal deformation coefficient of the quartz inclusions $\alpha_{q}$ was determined by least-square fitting the experimental measurements to the Schapery model. The elastic modulus $E_{q}$ and Poisson's ratio $\nu_{q}$ of quartz were taken, from the literature, as $73 \mathrm{GPa}$ and 0.17 , respectively [26]. Here, the resulting value of $\alpha_{q}$ was $3.9 \mu \varepsilon /{ }^{\circ} \mathrm{C}$. Note that a wide range of thermal deformation coefficient values for quartz are reported in the literature, ranging from $0.5 \mu \varepsilon /{ }^{\circ} \mathrm{C}$ for silica (amorphous) [27] to $9.3 \mu \varepsilon /{ }^{\circ} \mathrm{C}$ for quartzite [28].

\subsubsection{Mixed inclusion measurements}

Fig. $7 \mathrm{c}$ plots the experimentally measured effective thermal deformation coefficient $\alpha_{\text {eff }}$ of microencapsulated PCM-mortar composite specimens with both PCM and quartz sand inclusions as a function of PCM volume fraction $\phi_{c+s}$ ranging from 0 to 0.5 . Here, the total inclusion volume fraction was kept constant for all specimens such that $\phi_{q}+\phi_{c+s}=0.5$. In particular, note that $\alpha_{e f f}$ for a specimen with $\phi_{c}$ ${ }_{+s}=0.2$ and $\phi_{q}=0.3$ was measured to be $14.0 \mu \varepsilon /{ }^{\circ} \mathrm{C}$ while that of the cementitious matrix was measured earlier as $\alpha_{m}=14.1 \mu \varepsilon /{ }^{\circ} \mathrm{C}$. Thus, the effect of adding 20 vol.\% microencapsulated PCM was "offset" by the addition of 30 vol.\% quartz. Finally, Fig. 7c shows the predictions of $\alpha_{\text {eff }}$ by Schapery's model using the values of $\alpha_{c+s}$ and $\alpha_{q}$ retrieved from previous experiments (Fig. 7a-b). Excellent agreement was found between $\alpha_{\text {eff }}$ predicted by the Schapery model and measured experimentally. These results demonstrate the ability of Schapery's model to account for the combined, independent effects of both inclusions on the effective thermal deformation coefficient of the mortars.

\subsubsection{Design rule for thermal deformation equivalence in PCM-mortar composites}

As shown in Fig. 7, the addition of PCM microcapsules tends to increase the effective thermal deformation coefficient of the composite while the addition of quartz sand tends to decrease it. Therefore, one could "compensate" for the increase in $\alpha_{\text {eff }}$ caused by the addition of PCM microcapsules by adding an appropriate amount of quartz sand to maintain the ratio $\alpha_{e f f} / \alpha_{m}$ below some threshold. Fig. 8 plots the required quartz sand volume fraction $\phi_{q}$ corresponding to a ratio $\alpha_{e f f} /$ $\alpha_{m}$ equal to $1.25,1$, or 0.75 , as a function of microencapsulated PCM volume fraction $\phi_{c+s}$ ranging from 0 to 0.3 . Here, Schapery's model was used with the retrieved values of $\alpha_{c+s}$ and $\alpha_{q}$ to determine $\phi_{q}$ for each $\alpha_{e f f} / \alpha_{m}$ and $\phi_{c+s}$. Note that for a desired ratio $\alpha_{e f f} / \alpha_{m}<0.75$, PCM

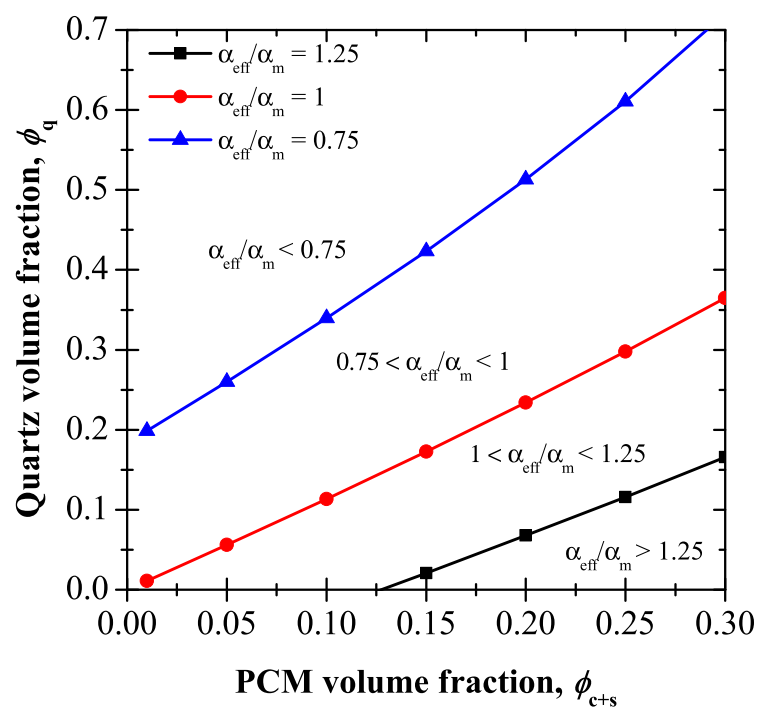

Fig. 8. Required quartz sand volume fraction $\phi_{q}$ to ensure that the ratio $\alpha_{e f f} / \alpha_{m}$ of a PCMmortar composite remains equal to $1.25,1$, or 0.75 , plotted as a function of microencapsulated PCM volume fraction $\phi_{c+s}$ ranging from 0 to 0.3 . 
volume fractions above $\sim 0.25$ are not feasible as they require a minimum total inclusion volume fraction $\phi_{c+s}+\phi_{q}$ of 0.85 or higher. Also, for an allowable ratio of $\alpha_{\text {eff }} / \alpha_{m}=1.25$, no quartz sand is required for PCM volume fraction $\phi_{c+s}$ below 0.125 .

\section{Conclusion}

This paper examined the effect of microencapsulated PCMs and other particulate inclusions on the thermal deformation behavior of cementitious composites, and presented a convenient approach to estimate the thermal deformation coefficient of inclusions based on measured effective properties. The effective thermal deformation coefficient of three-component core-shell-matrix composites was first predicted numerically using finite element simulations of free thermal deformation. It was found to be a function of only the constituents' volume fractions and thermomechanical properties. Predictions from the effective medium approximation developed by Schapery were found to agree well with the numerical results over a wide range of constituent thermal deformation coefficients. Moreover, the effective thermal deformation coefficient of cementitious microencapsulated PCM-composites was measured experimentally for cement paste and cement mortar with various volume fractions of quartz sand and microencapsulated PCM. The experimental measurements were used in conjunction with Schapery's model to retrieve the thermal deformation coefficient of PCM microcapsules and of quartz sand grains. The thermal deformation coefficient of the PCM microcapsules was found to be near that of the shell material, due to the fact that the capsules might not be completely filled with PCM, thus leaving space inside for the PCM core to expand. Schapery's model was shown to predict accurately the effective thermal deformation coefficient of cementitious composite samples with a mixture of microencapsulated PCM and quartz sand inclusions based on the previously retrieved properties. Finally, a design rule was suggested for determining the amount of quartz sand required to offset the effect of the PCM microcapsules in increasing the thermal deformation coefficient of PCM-mortar composites. These results could be useful in modeling the thermal deformation behavior of pavement sections featuring microencapsulated PCMs for crack resistance.

\section{Acknowledgments}

The authors acknowledge the financial support for this work provided by an Infravation ERA-NET Plus grant (31109806.0001: ECLIPS), National Science Foundation (CMMI:1130028, CAREER: 1253269) and California Energy Commission (contract: PIR: 12-032). The authors acknowledge the financial support provided by The Sustainable L.A. Grand Challenge and the Office of the ViceChancellor for Research at UCLA. The contents of this paper reflect the views and opinions of the authors, who are responsible for the accuracy of the datasets presented herein, and not necessarily the views of the funding organizations. The Laboratory for the Chemistry of Construction Materials $\left(\mathrm{LC}^{2}\right.$ ) and the Molecular Instrumentation Center at UCLA acknowledge the support that has made their operations possible.

\section{References}

[1] A.W. Herrmann, ASCE 2013 Report Card for America's Infrastructure, IABSE Symposium Report, 99 International Association for Bridge and Structural Engineering, 2013, pp. 9-10.

[2] S. Wu, D. Huang, F. Lin, H. Zhao, P. Wang, Estimation of cracking risk of concrete at early age based on thermal stress analysis, J. Therm. Anal. Calorim. 105 (1) (2011) $171-186$.

[3] J. Tanesi, M. Kutay, A. Abbas, R. Meininger, Effect of coefficient of thermal expansion test variability on concrete pavement performance as predicted by mechanistic-empirical pavement design guide, J. Transp. Res. Board 2020 (2007) $40-44$.

[4] D.P. Bentz, R.J. Turpin, Potential applications of phase change materials in concrete technology, Cem. Concr. Compos. 29 (2007) 527-532.

[5] F. Fernandes, S. Manari, M. Aguayo, K. Santos, T. Oey, Z. Wei, G. Falzone, N. Neithalath, G. Sant, On the feasibility of using phase change materials (PCMs) to mitigate thermal cracking in cementitious materials, Cem. Concr. Compos. 51 (2014) 14-26.

[6] L.F. Cabeza, Advances in Thermal Energy Storage Systems: Methods and Applications, Elsevier, Cambridge, UK, 2014.

[7] M.R. Eslami, R.B. Hetnarski, J. Ignaczak, N. Noda, N. Sumi, Y. Tanigawa, Theory of Elasticity and Thermal Stresses, Springer, New York, NY, 2013.

[8] K.D. Hjelmstad, Fundamentals of Structural Mechanics, second edition, Springer, New York, NY, 2005.

[9] W. Voigt, Uber die beziehung zwischen den beiden elastiziatskonstanten isotroper korper, Ann. Phys. 274 (12) (1889) 573-587.

[10] P.S. Turner, Thermal expansion stresses in reinforced plastics, J. Res. Natl. Bur. Stand. 37 (1946) 239.

[11] R.A. Schapery, Thermal expansion coefficients of composite materials based on energy principles, J. Compos. Mater. 2 (3) (1968) 380-404.

[12] Z. Hashin, Analysis of composite materials, J. Appl. Mech. 50 (2) (1983) 481-505.

[13] A.S.T.M. Standard, "C150", Standard Specification for Portland Cement, ASTM International, West Conshohocken, PA, USA, 2016.

[14] A.S.T.M. Standard, "C192", Standard Practice for Making and Curing Concrete Test Specimens in the Laboratory, ASTM International, West Conshohocken, PA, USA, 2016.

[15] A.S.T.M. Standard, "C778", Standard Specification for Standard Sand, ASTM International, West Conshohocken, PA, USA, 2016.

[16] A.S.T.M. Standard, "C157", Standard Test Method for Length Change of Hardened Hydraulic-Cement Mortar and Concrete, ASTM International, West Conshohocken, PA, USA, 2014.

[17] D.J. Janssen, M.B. Snyder, Resistance of Concrete to Freezing and Thawing, Strategic Highway Research Program, National Research Council, 1994.

[18] A. Kumar, T. Oey, S. Kim, D. Thomas, S. Badran, J. Li, F. Fernandes, N. Neithalath, G. Sant, Simple methods to estimate the influence of limestone fillers on reaction and property evolution in cementitious materials, Cem. Concr. Compos. 42 (2013) 20-29.

[19] A.M. Thiele, A. Kumar, G. Sant, L. Pilon, Effective thermal conductivity of threecomponent composites containing spherical capsules, Int. J. Heat Mass Transf. 73 (2014) 177-185.

[20] B.A. Young, A.M.K. Fujii, A.M. Thiele, G. Sant, L. Pilon, Effective elastic moduli of core-shell-matrix composites, Mech. Mater. 92 (2016) 94-106.

[21] S. Kim, H. Moon, J. Kim, Thermal characterizations of the paraffin wax/low density polyethylene blends as a solid fuel, Thermochim. Acta 613 (2015) 9-16.

[22] W. Martienssen, H. Warlimont, Springer Handbook of Condensed Matter and Materials Data, Springer Science \& Business Media, New York, NY, 2006.

[23] J.M. Gere, B.J. Goodno, Mechanics of Materials, Nelson Education, Scarborough, ON, 2012.

[24] S. Walker, D.L. Bloem, W.G. Mullen, Effects of temperature changes on concrete as influenced by aggregates, Journal Proceedings of the American Concrete Institute, 48 1952, pp. 661-679.

[25] D.J. Hart, H.F. Wang, Laboratory measurements of a complete set of poroelastic moduli for berea sandstone and Indiana limestone, J. Geophys. Res. Solid Earth 100 (B9) (1995) 17741-17751.

[26] L.L.C. MatWeb, MatWeb material property data, (2013) http://www.matweb.com Accessed: Oct 2016.

[27] J. Oishi, T. Kimura, Thermal expansion of fused quartz, Metrologia 5 (2) (1969) 50.

[28] K. Hall, S. Tayabji, Coefficient of thermal expansion in concrete pavement design, Tech. Rep. 2011. 